\title{
Eco-design e sostenibilità nella nautica da diporto: il caso della Regione Marche*
}

\author{
TONINO PENCARELLI ${ }^{* *}$ SIMONE SPLENDIANI ${ }^{* * *}$ MaURO DINI ${ }^{* * * *}$
}

\begin{abstract}
Obiettivo del paper: Obiettivo dello studio è valutare il grado di orientamento alla sostenibilità delle imprese appartenenti al sistema del valore della nautica da diporto della Regione Marche, sia in termini di consapevolezza strategica che di effettive politiche operative attuate.

Metodologia: Lo studio è stato effettuato applicando un modello concettuale volto a valutare l'orientamento alla sostenibilità (sustainability orientation) delle imprese osservate, attraverso un'indagine prettamente qualitativa. La raccolta dati è stata effettuata attraverso differenti canali: interviste semi-strutturate e analisi documentale.

Risultati: Dall'indagine emerge come le imprese terminali (ovvero quelle a contatto con il cliente finale) si dimostrino maggiormente consapevoli rispetto ai temi della sostenibilità delle imprese che operano a monte della filiera, potendo essere quindi definite come imprese sustainability oriented secondo il modello teorico di riferimento. Lo studio mostra la necessità di una maggiore integrazione delle imprese di fase nei processi di eco-design.

Limiti della ricerca: I maggiori limiti ascrivibili al lavoro riguardano la limitatezza del campione, ovvero l'impossibilità di compiere generalizzazioni, oltre che la mancanza di un'analisi longitudinale, volta a cogliere i cambiamenti dei comportamenti strategici nel tempo.

Implicazioni pratiche: Il successo competitivo nella nautica da diporto dei prossimi anni dovrà fondarsi, oltre che sulle tradizionali fonti di vantaggio competitivo del settore, sulla capacità di realizzare imbarcazioni attente all'eco-compatibilità ed alla minimizzazione dell 'impatto ambientale in ogni fase del suo ciclo vita.

Originalità del lavoro: L'originalità del paper risiede nel proporre un modello sintetico di analisi del grado di orientamento alla sostenibilità delle imprese della nautica,
\end{abstract}

* Sebbene il paper sia il frutto di un lavoro condiviso, sono da attribuire a Splendiani S. e a Dini M. i paragrafi 1 e 2, a Pencarelli T. e Dini M. il paragrafo 3 e a tutti gli autori il paragrafo 4 .

** $\quad$ Ordinario di Economia e Gestione delle Imprese - Università degli Studi di Urbino e-mail: tonino.pencarelli@uniurb.it

*** Ricercatore di Economia e Gestione delle Imprese - Università degli Studi di Perugia e-mail: simone.splendiani@unipg.it

**** Dottorando di ricerca in Economia e Management - Università degli Studi di Urbino e-mail: mauro.dini@uniurb.it 
comprensivo del concetto di eco-design, per uno sviluppo sostenibile che coinvolga l'intero sistema del valore della nautica da diporto.

Parole chiave: nautica da diporto; eco-design; sviluppo sostenibile; orientamento alla sostenibilità; vantaggio competitivo; innovazione

Purpose of the paper: The aim of this study was to evaluate the degree of orientation to the sustainability of enterprises belonging to the yachting value system in the Marche Region, both in terms of strategic awareness that in terms of operational policies implemented.

Methodology: The study was carried out by applying a conceptual model designed to assess the sustainability orientation of enterprises, observed through a survey purely qualitative. The data collection was conducted through different channels: semi-structured interviews and documentary analysis.

Findings: The survey shows how end-user sales companies prove to be more aware with respect to sustainability issues than subcontractors and may be then defined as "sustainability oriented" enterprises, according to the theoretical model.

Research limitations: The main limitations are the narrowness of the sample, namely the inability to make generalizations, as well as the lack of a longitudinal analysis, aimed at seizing the changes of strategic behavior over time.

Implications: In the future, the competitive success in the business of recreational boating must be based not only on traditional sources of competitive advantage, but also on the ability to achieve eco-compatibility boats capable of minimizing environmental impact at every stage of its life cycle.

Originality of the paper: The originality of the paper lies in proposing a synthetic model of analysis of the degree of the sustainability orientation of the yachting companies including the concept of eco-design.

Key words: yachting industry; eco-design; sustainable development; sustainability orientation; competitive advantage; innovation

\section{Caratteri e tendenze evolutive del settore della nautica da diporto in Italia e nella Regione Marche}

Negli anni antecedenti la crisi economica del 2008 il comparto della nautica da diporto ha registrato, in Italia, una crescita costante e vigorosa, consentendo alle imprese nostrane di competere con i principali player mondiali e di conquistare la leadership in alcune nicchie di mercato.

Tuttavia, la crisi ha stravolto le dinamiche competitive, sia di livello internazionale che locale. Secondo i dati UCINA il fatturato complessivo del settore in Italia è passato da 4.986 milioni di euro nell'anno 2007 a 2.080 milioni di euro nel corso del 2012, segnando una contrazione del $58 \%{ }^{1}$.

1 Secondo alcuni autori (Lazzaretti e Capone, 2009) il comparto in Italia risulta caratterizzato da forme oligopolistiche, ovvero dalla presenza di grandi player a contatto diretto con il mercato finale e quindi capaci di indirizzare le politiche produttive di una 
La regione Marche, ambito di riferimento per il nostro studio, risulta tra i sistemi più colpiti dalla crisi. Infatti, se nel 2007 il numero di imprese marchigiane sul totale di quelle italiane rappresentava il 3\%, nel 2011 solamente lo 0,6\% (Fig.1) ${ }^{2}$.

Fig. 1: Comparto della nautica da diporto, distribuzione percentuale delle aziende nelle principali regioni italiane (anni 2008/2011)

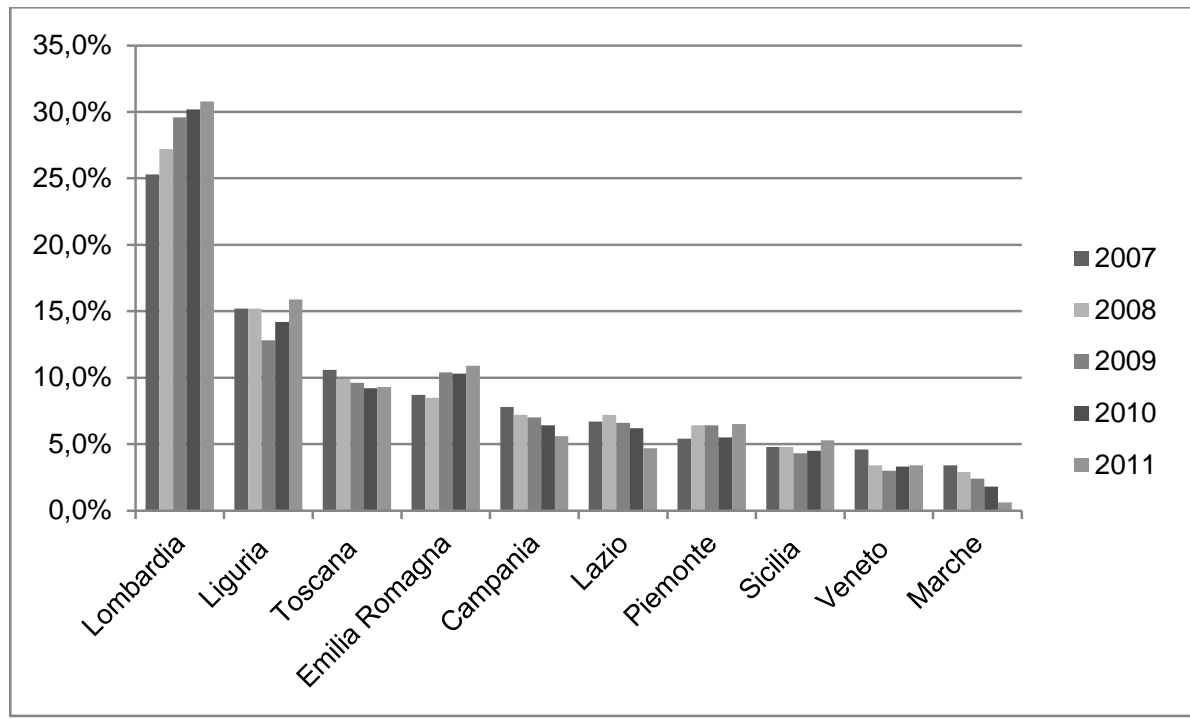

Fonte: UCINA (2008; 2009; 2010;2011, 2012)

Un tracollo simile è stato registrato nel numero di addetti, passati dal 5,7\% sul totale italiano nel 2007 all'1,4\% del 2011 (Fig. 2).

numerosa serie di piccole imprese di fase. Tre sarebbero dunque le categorie di imprese individuate in tal senso, rispecchiando altresì il sistema marchigiano ed offrendo una prima suddivisione tipologica ripresa poi nella ricerca empirica (Tracogna, 2010):

1) pochi costruttori di grandi dimensioni, con elevato orientamento alle esportazioni (imprese terminali);

2) alcuni cantieri di piccola-media dimensione, specializzati in nicchie di alta qualità (imprese di fase);

3) ampio sistema di piccole imprese, prevalentemente artigiane che completano la filiera locale della nautica e che operano come sistema di indotto dei grandi cantieri (imprese di fase).

Giova peraltro sottolineare come tale configurazione risulti essere del tutto simile a quella riscontrabile nella regione Marche (Fortezza, 2008) consentendo di estendere le riflessioni e i risultati dello studio al di là dei confini regionali.

2 Per un'analisi della competitività e dei risultati economico-finanziari delle imprese della nautica di diporto vedi Ferrando et al., 2014. 
Fig. 2: Comparto della nautica da diporto, distribuzione percentuale degli addetti nelle principali regioni italiane (anni 2008/2011)

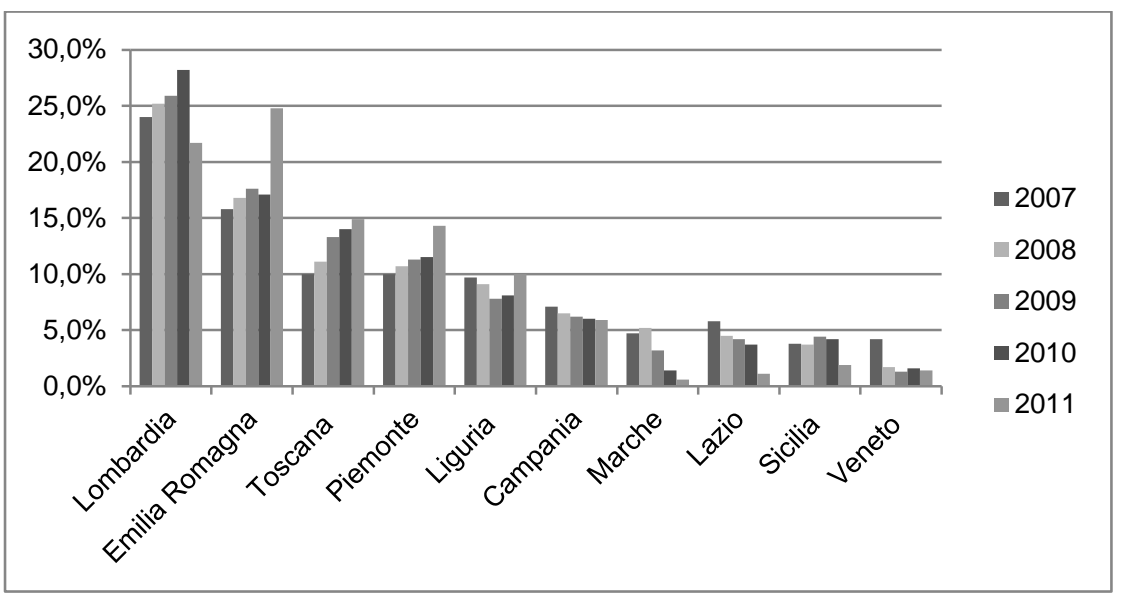

Fonte: UCINA (2008; 2009; 2010; 2011; 2012)

Tali evidenze lasciano emergere una situazione di declino strutturale dovuta non solo alla crisi globale, ma anche alla perdita di competitività delle imprese marchigiane nel contesto nazionale e delle imprese italiane in generale. In tal senso, diversi autori hanno sottolineato come risulti necessaria, da un lato, una migliore conoscenza delle dinamiche della domanda da parte dei produttori e, dall'altro, l'attuazione di politiche per l'innovazione di prodotto e di processo, stimolate da interventi di politica industriale a livello nazionale ed europeo (Tracogna, 2007, 2010; Nobili, 2012; Tola, 2013). La via dell'innovazione - fondata su competenze tecniche di alto livello - consente alle imprese di offrire prodotti a maggior valore aggiunto per acquirenti particolarmente sensibili all'originalità, al design, all'estetica, al confort, alla qualità dei materiali ed alle prestazioni delle imbarcazioni. In particolare, negli ultimi anni hanno assunto un ruolo centrale le innovazioni di prodotto e di processo caratterizzate dall'eco-compatibilità, attente cioè all'impatto ambientale in ogni fase del ciclo di vita dell'imbarcazione (Bruni e Carcano, 2009; Moschini, 2009).

Tale tendenza - secondo il Rapporto GreenItaly $(2010,2011,2012)$ - sembra già emersa all'interno del sistema di offerta, comportando una rimodulazione dell'impostazione manageriale su nuovi fattori critici di successo legati alla sostenibilità, da accostare a quelli tradizionali quali la tecnologia, l'estetica e la funzionalità dei prodotti. La sostenibilità dei prodotti, in altri termini, può rappresentare un fattore di differenziazione in grado di costruire una nuova fonte di vantaggio competitivo sui mercati internazionali.

Lo studio sviluppato si inserisce in tale prospettiva, avendo lo scopo di valutare il grado di orientamento alla sostenibilità delle imprese del comparto operanti nella 
regione Marche, al fine di comprenderne le potenzialità future di rilancio della competitività attraverso tali percorsi innovativi.

Nello specifico, la ricerca empirica è articolata nelle seguenti domande di ricerca:

- Qual è il grado di consapevolezza delle imprese rispetto al tema dello sviluppo sostenibile? (Dimensione culturale) (\$3.3.1)

- Quali politiche rivolte alla sostenibilità vengono adottate? (Dimensione comportamentale) (\$3.3.2)

- Qual è l'impatto sulla gestione strategica delle politiche adottate? (\$3.3.3)

\section{L'eco-design come prospettiva culturale e strategica delle imprese della nautica da diporto}

I problemi legati all'ambiente e alla sostenibilità delle attività turistiche stanno ormai da tempo attirando l'attenzione di organi legislativi di ogni ordine e grado, dagli enti locali all'Unione Europea (Antonelli et al., 2005). Quest'ultima, in particolare, ha prodotto negli ultimi anni una copiosa legislazione in tal senso, con importanti ripercussioni anche nel settore della nautica da diporto, seppur non si registri ad oggi uno specifico ed organico impianto normativo di riferimento per il comparto stesso ${ }^{3}$.

Attraverso il documento approvato dalla commissione europea nel 2010, "Una strategia per una crescita intelligente, sostenibile e inclusiva", infatti, l'Unione Europea propone misure concrete in materia di energia e cambiamenti climatici che fissano nuovi obiettivi per il 2020, condividendo l'obiettivo ritenuto strategico dalla comunità scientifica di impedire che nel 2050 l'aumento della temperatura atmosferica superi i $2{ }^{\circ} \mathrm{C}$.

La strategia adottata mira ad indirizzare la società europea verso un modello di sviluppo economico sostenibile, promuovendo un'economia a basse emissioni di $\mathrm{CO} 2$, attraverso un ampio ventaglio di strumenti e regole, tra i quali: la Politica Integrata di Prodotto (Integrated Product Policy - IPP), i requisiti di eco-design (Energy-using Products - EuP $P^{4}$, la gestione dei rifiuti di apparecchiature elettriche ed elettroniche (Waste of Electric and Electronic Equipmente - WEEE), il sistema comunitario di ecogestione e audit (Eco-Management and Audit Scheme - EMAS), la direttiva 2003/44/CE (riguardante le disposizioni legislative, regolamentari e amministrative delle imbarcazioni da diporto) e la recente direttiva 2013/53/EU sulle unità da diporto.

3 Va tuttavia menzionata la presenza di associazioni e società di riferimento per le norme e certificazioni: Marittime Coastguard Agency (MCA), l'International Marittime Organization (IMO) e RINA.

4 La prima misura attuativa della Direttiva EuP è l'implementazione della modalità stand by/off: regolamento adottato dalla Commissione Europea nel dicembre del 2008 ed è entrato in vigore il 7 gennaio 2009. 
Sebbene i vari dispositivi non si riferiscano direttamente alla produzione di imbarcazioni da diporto (esclusa le direttive 2003/44/CE e 2013/53/EU), essi mirano alla diffusione del concetto di progettazione ecocompatibile (Vezzoli e Manzini, 2007; Thackara, 2008; Tamborrini, 2008; Di Maria e Bettiol, 2009; Zuccon, 2009), secondo un approccio dinamico ed olistico riassunto dal termine "Eco-design". Trattasi di una filosofia di produzione capace di andare oltre la mera riduzione dell'impatto ambientale del prodotto, orientando piuttosto le politiche aziendali verso la creazione di un "contesto di forte interazione fra scoperta scientifica, applicazione tecnologica, buon disegno ed effetto sociale positivo" (Koenig, 1983, pp. 24). Giova sottolineare la rilevanza della dimensione sociale, intesa non solo come tutela della comunità locale (Benevolo, 2011), ma anche come l'implementazione di processi innovativi a carattere culturale e tecnologico in grado di garantire la massima accessibilità alle persone diversamente abili.

Il concetto di eco-design, ampio e articolato, può essere a nostro giudizio scomposto secondo tre dimensioni principali, graficamente rappresentate nella figura 3:

1) sul piano dell' ergonomia: allude alla capacità di valorizzare l'interazione fra gli individui e la tecnologia, che permetta di vivere il rapporto con la natura e in particolare con il mare, rendendo la barca un piacevole continuum con la terra ferma (Zuccon, 2009);

2) sul piano del profilo estetico: si riferisce ad alte prestazioni nel design, con una ricerca stilistica attenta alle nuove tendenze cercando di realizzare un carattere di "unicità del prodotto" (Nobili, 2012) ;

3) sul piano dell'innovazione tecnologica (Tracogna, 2007; 2010): si rivolge alla dimensione della sostenibilità, declinata secondo le seguenti prospettive:

- design volto alla riduzione di risorse utilizzate: limitare i materiali usati per la produzione e utilizzo di prodotti e servizi eco-efficenti a parità di performance;

- design rivolto al risparmio energetico: miglioramento dell'efficienza energetica dei prodotti;

- design modulare per il riciclaggio: design rivolto al riciclaggio dei materiali utilizzati e allo smontaggio più semplice al termine del ciclo di vita del prodotto;

- design "for all": progettazione degli spazi e servizi per le esigenze delle persone diversamente abili.

Fig. 3: La dimensione concettuale dell' eco-design

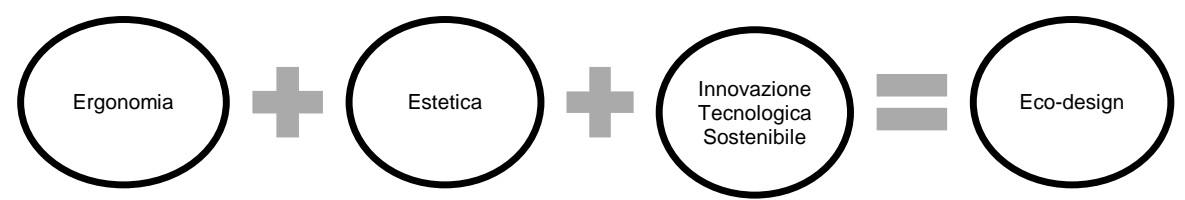

Fonte: nostra elaborazione 
L'attenzione ai contenuti della sostenibilità nei prodotti del comparto della nautica da diporto emerge tuttavia anche dal lato della domanda, soprattutto da parte di nicchie di mercato costituite da utilizzatori esperti, affascinati dall'esplorare nuove strade con uno stile più sobrio e semplice, che permetta di vivere la forte relazione fra uomo e natura (Pencarelli e Forlani 2007; Benevolo, 2010).

Tali tendenze - secondo alcuni - risultano essere particolarmente influenzate da fattori contingenti, come il crescente costo dei combustibili fossili, gli effetti della crisi economica che ha ridotto il potere di acquisto dei consumatori (Bruni e Carcano, 2009; Quildon e Pun, 2011; GreenItaly, 2012), i divieti imposti dalle autorità locali in merito all'accesso di imbarcazioni inquinanti in alcune zone esclusive (Moschini, 2009) e, non ultimo, l'affermazione e il diffondersi di una ethical mind degli individui verso comportamenti dannosi per l'ambiente (Gardner, 2007).

In questa prospettiva, la capacità delle imprese del comparto di produrre secondo logiche di eco-design si sostanzierebbe in innovazioni progettuali orientate al miglioramento delle performance e del confort a bordo, al risparmio energetico, all'ergonomia degli scafi per rendere più piacevole la navigazione, all'uso di materiali non inquinanti, a motori efficienti e poco rumorosi, oltre che alla crescente attenzione ad attività fino a pochi anni fa non considerate come lo smaltimento sostenibile delle imbarcazioni stesse (Grande, 2009). Questo anche nell'ottica di un posizionamento da first mover di fronte a probabili nuove regole europee a favore di produzioni ecologiche, aggiungendo alla tradizionale gamma di imbarcazioni, anche modelli concepiti per la nicchia di domanda interessata alla sostenibilità .

Una produzione nautica che si orienti alla sostenibilità, quindi, riconfigurerebbe l'intero value system a partire dalla revisione del sistema di offerta, per poi diffondersi tra le varie imprese coinvolte nel processo di value creation.

Tale ri-orientamento risulta assai complesso e ricco di ostacoli, a causa di fattori strutturali come la debole consapevolezza strategica delle imprese e il forte sbilanciamento del potere contrattuale a favore dei cantieri terminali (Nobili, 2012). L'elemento chiave per una ricostruzione in senso sostenibile dell'industria nautica pare dunque essere l'orientamento alla sostenibilità delle singole imprese in essa operanti, potenzialmente foriero di innovazioni di prodotto e di processo capaci di accrescere il valore percepito e consegnato ai consumatori. In tale prospettiva e secondo tale logica si sviluppa l'analisi empirica di seguito riportata.

\section{Analisi empirica}

\subsection{Framework concettuale e metodologia}

Obiettivo dello studio è valutare il grado di orientamento alla sostenibilità di un gruppo di imprese nautiche marchigiane. Il modello teorico di riferimento (Pencarelli e Splendiani, 2012), illustrato nella Figura 4, si compone essenzialmente di due dimensioni: la prima inerente la cultura imprenditoriale e la seconda quella 
dei comportamenti effettivamente praticati dall'impresa o dalle imprese oggetto di valutazione.

La dimensione culturale attiene ai principi e alle credenze dichiarate dall'imprenditore e mira a cogliere la predisposizione all'attuazione di politiche orientate alla sostenibilità. Ad una prima analisi tale dimensione potrebbe essere intesa come propedeutica ad un effettivo impegno in politiche sostenibili, ma il rapporto tra le due risulta invece assai meno lineare e deterministico. Le variabili indicative di tale dimensione sono la consapevolezza circa il significato stesso di orientamento alla sostenibilità e la propensione all'investimento in politiche sostenibili.

La dimensione comportamentale, invece, mira a valutare l'effettiva applicazione di politiche per la sostenibilità, declinate su tre livelli:

- adozione di condotte finalizzate all'ottenimento di certificazioni ambientali e di qualità, le quali, oltre ai benefici nella gestione operativa dell'impresa, hanno l'obiettivo di rafforzare il corporate brand nei confronti degli stakeholders (bilancio sociale, certificazioni e partnership, ecc.);

- comportamenti virtuosi non certificati, ovvero le misure adottate all'interno dell'impresa in favore di uno sviluppo sostenibile (dotazione tecnologica per risparmio energetico e idrico, produzione energia da fonti rinnovabili, strumenti per la raccolta differenziata, ecc.)

- sviluppo di prodotti e servizi di eco-design: progettazione e sviluppo di prodotti eco-compatibili, con coinvolgimento di tutti gli attori della supply chain e del value system.

Fig. 4: Le componenti dell'orientamento alla sostenibilità delle imprese della nautica da diporto

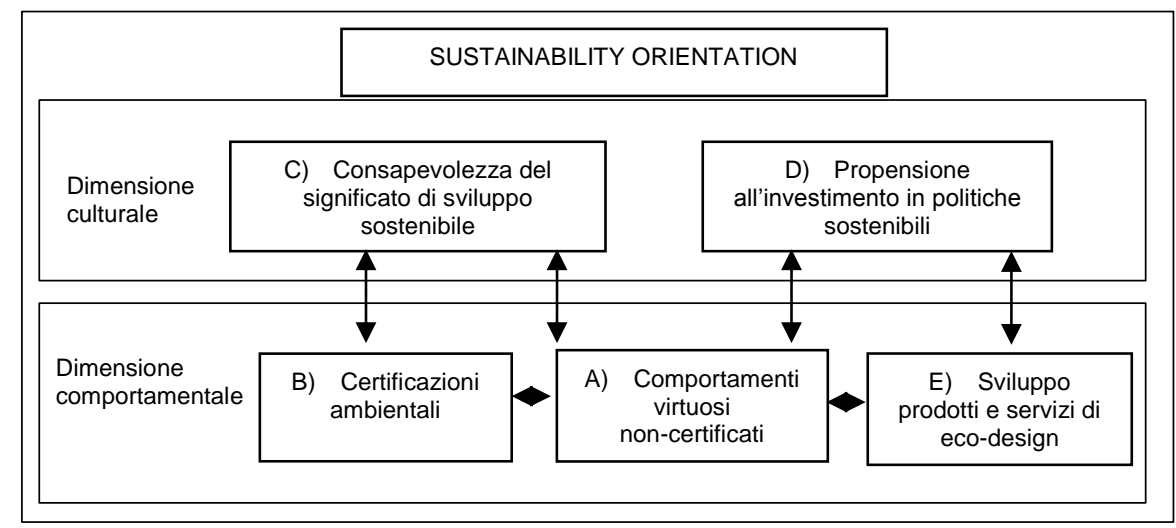

Fonte: adattamento da Pencarelli e Splendiani, 2012

La ricerca ha richiesto la raccolta di una rilevante quantità di dati, attraverso tre diversi - ma integrati - piani di analisi (Yin, 2009). 
Il primo è l'intervista semi-strutturata (Corbetta, 1999) rivolta a 30 imprese $^{5}$ di settore con sede legale o operativa nella Regione Marche ${ }^{6}$. Il campionamento adottato è di tipo non probabilistico, ovvero un campionamento ragionato ${ }^{7}$, attraverso la quale si è cercato di rappresentare l'intero universo della nautica da diporto, bilanciando le imprese terminali con quelle di fase.

Un secondo strumento è l'intervista semi-strutturata rivolta a due esponenti di associazioni di categoria ${ }^{8}$ e al responsabile scientifico del progetto ENA. Come ogni intervista non strutturata, i colloqui non hanno seguito un set di domande prestabilite, ma una traccia indicativa che mettesse in luce la relazione fra politiche sostenibili e imprese.

Infine si è ricorso all'analisi documentale (Corbetta, 1999), attraverso i siti internet delle imprese intervistate al fine di approfondire alcuni aspetti emersi dalle interviste.

Il mix degli strumenti prescelti (interviste qualitative non strutturate e analisi dei siti), è sembrato il migliore ai fini dell'indagine, avendo la ricerca l'obiettivo di cogliere gli elementi della gestione strategica dell'impresa nei confronti delle politiche sostenibili.

I maggiori limiti ascrivibili al lavoro riguardano la limitatezza del numero di imprese indagate, oltre alla mancanza di un'analisi longitudinale volta a cogliere i cambiamenti dei comportamenti strategici nel tempo.

\subsection{Le imprese oggetto di analisi}

Il gruppo di aziende sotto indagine è costituito da 5 imprese terminali e 25 imprese di fase. Nel $66 \%$ dei casi il fatturato non supera i due milioni di euro, il $17 \%$ delle imprese si posiziona tra 2 e 10 milioni di euro, mentre il rimanente $17 \%$ del campione fattura oltre 10 milioni di euro.

Con riferimento al numero di addetti, il $69 \%$ delle aziende intervistate dichiara di avere meno di 10 dipendenti, il 17\% si posiziona nella classe tra 11-50 dipendenti, mentre solo il $14 \%$ afferma di superare i 50 dipendenti.

Le aziende che si posizionano nelle fasce più alte (per fatturato e dipendenti) sono le imprese terminali, mentre le imprese di fase si concentrano per la quasi totalità dei casi nelle fasce più basse.

Le imprese di fase realizzano la quasi totalità del fatturato sul mercato nazionale

5 Le interviste fanno parte dello studio dei "fabbisogni formativi delle imprese della nautica da diporto nella regione Marche" effettuate nel corso del Progetto ENA.

6 Nel caso di una impresa terminale è stato ritenuto sufficiente la localizzazioni di siti produttivi nella Regione Marche.

7 In questo tipo di campionamento le unità vengono scelte dal ricercatore senza procedure casuali, in base ad un ragionamento (in sostanza uno o più criteri).

8 In particolare, gli intervistati sono stati: Francesco Buscaglia e Valeria Bertani (Confindustria Pesaro e Urbino), Moreno Bordoni (CNA Pesaro e Urbino), Silvana Della Fornace (Confartigianato Pesaro e Urbino) e infine a Ezio Businaro (responsabile scientifico progetto ENA). Le interviste sono state effettuate nel gennaio 2013. 
$(88,1 \%)$, mentre le imprese terminali conseguono buona parte del fatturato all'estero $(76,6 \%)$.

\subsection{Risultati}

\subsubsection{Dimensione culturale}

Per quanto concerne la dimensione culturale, nelle interviste sono state poste due differenti domande: la prima per valutare se gli intervistati sono consapevoli del significato di sviluppo sostenibile, attraverso la selezione di quattro variabili fra una serie proposta (Figura 5).

Fig. 5: Domanda del questionario: quali fra le seguenti variabili sono collegate al tema dello sviluppo sostenibile?

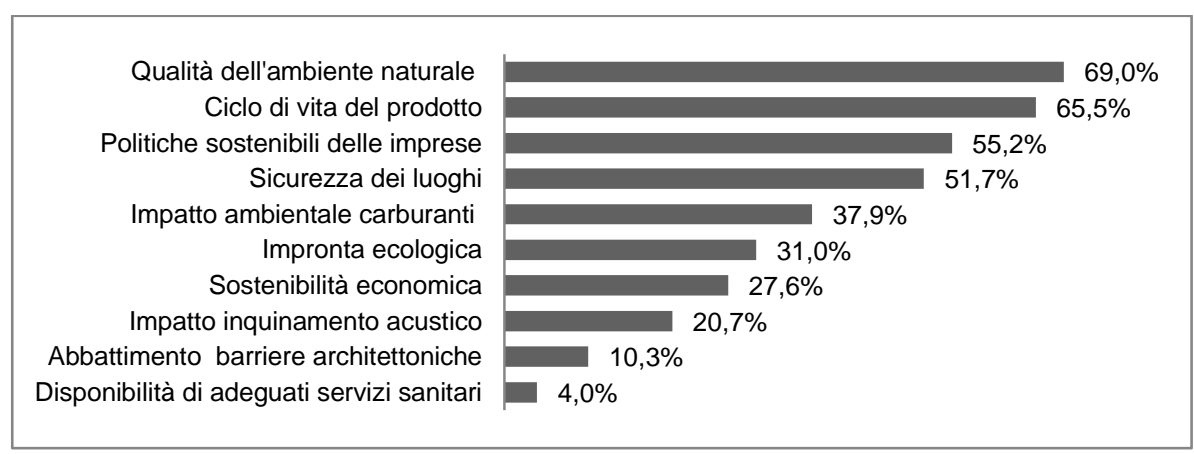

Fonte: nostra elaborazione

Le variabili che presentano una frequenza maggiore sono: qualità dell'ambiente naturale $(69,0 \%)$, valutazione dell'intero ciclo di vita dell'imbarcazione $(65,5 \%)$, impegno da parte delle imprese in politiche rivolte alla sostenibilità $(55,2 \%)$ e sicurezza dei luoghi $(51,7 \%)$.

Quasi tutti gli imprenditori comprendono il significato di sviluppo sostenibile e lo interpretano principalmente attraverso la prospettiva ambientale.

Nella seconda domanda si è cercato di valutare la propensione o la volontà di effettuare investimenti rivolti allo sviluppo sostenibile.

La maggior parte degli intervistati $(75 \%)$ considera importante pianificare strategie future rivolte alla sostenibilità, il $22 \%$ non ha una chiara posizione sul tema collocandosi nella categoria "non so", mentre solo un intervistato dichiara che non vale la pena investire in tali politiche.

Tra le motivazioni di coloro che ritengono importante investire in politiche rivolte alla sostenibilità, emerge che l'opzione più selezionata è "perché produce benefici per il futuro" (54,2\%), a seguire "perché è un obbligo morale" (29,2\%) e infine "perché è quello che vuole il mercato" (16,8\%). 
Dall'analisi delle diverse tipologie d'impresa che rispondono positivamente non emergono particolari differenze.

Emerge nel complesso una buona consapevolezza degli intervistati circa il significato di sviluppo sostenibile e delle sue possibili implicazioni nel comportamento dell'impresa. Tuttavia, tale sensibilità ai temi che riguardano la sostenibilità sembra legarsi maggiormente al tema dello sviluppo sostenibile globale e non all'effettiva esistenza di una nuova domanda di mercato green.

\subsubsection{Dimensione comportamentale}

Il secondo livello di analisi riguarda la dimensione comportamentale e si basa su tre differenti ambiti di osservazione.

Il primo prende in considerazione l'ottenimento di certificazioni ambientali e di qualità da parte delle imprese intervistate.

Fra le 30 imprese intervistate, solo 6 dichiarano di possedere certificazioni ambientali; tra queste 3 sono imprese terminali e 3 di fase.

La maggior parte delle imprese non certificate (22 casi su 25) sono quelle di fase, mentre 3 imprese terminali su 5 dichiarano di avere almeno una certificazione ambientale. Il diverso comportamento delle imprese terminali rispetto a quelle di fase si può spiegare con il fatto che mentre le prime utilizzano la certificazione ambientale anche come veicolo di comunicazione di mercato, cercando di intercettare i segmenti di domanda attenti e sensibili alle problematiche ecologiche e della sostenibilità, le imprese di fase non vedono vantaggi diretti e tangibili dall'adozione di queste pratiche di certificazione.

Le certificazioni conseguite riguardano per questo in misura maggiore i temi della qualità di processo e di prodotto e sono nella quasi totalità dei casi gli unici strumenti utilizzati: cinque imprese dichiarano di aver ottenuto l'ISO $9001 \mathrm{e}$ in singoli casi ISO 14001, EMAS e OHSAS 18001.

Un secondo aspetto indagato riguarda le pratiche virtuose non certificate, chiedendo agli intervistati di indicare quali, tra le seguenti opzioni, vengono applicate nell'attività ordinaria dell'impresa:

- inserimento di strumenti tecnologici per il risparmio idrico $(6,6 \%)$;

- inserimento strumento tecnologici per risparmio energetico $(10,0 \%)$;

- introduzione strumenti raccolta differenziata $(73,3 \%)$;

- utilizzo fonti energetiche rinnovabili $(13,3 \%)$;

- acquisti verdi $(6,6 \%)$.

Solo gli strumenti per la raccolta differenziata $(73 \%)$ sono presenti in misura significativa, mentre le altre opzioni presentano una debole intensità. Fra le diverse tipologie di impresa non emergono particolari differenze.

Per quanto riguarda il terzo profilo di analisi, vale a dire lo sviluppo di prodotti di eco-design, solo 7 aziende (il 23\% del campione) dichiarano di aver già attivato processi di questo tipo.

Da un'analisi qualitativa delle due tipologie di imprese in esame, emerge come fra le imprese terminali 3 imprese su 5 abbiano già attivato tali progetti, mentre fra 
le imprese di fase solo 4 imprese su 25 hanno attivato innovazioni guidate dai processi di eco-design, ed in particolare terzisti fornitori di attività di progettazione.

Per comprendere e approfondire quali fossero le caratteristiche dei prodotti di eco-design richiamati in figura 3 e sui quali le imprese terminali si stanno orientando, è stata effettuata un'analisi dei siti internet delle imprese che hanno risposto positivamente alla domanda.

È risultato che solo nei siti di tre imprese terminali si sono riscontrate informazioni rivolte a descrivere le caratteristiche dei prodotti di eco-design, mettendo in luce una maggiore attenzione alla comunicazione di mercato volta a rappresentare una maggiore sensibilità ai temi della sostenibilità.

Dall'analisi dei siti sono emersi elementi innovativi che riguardano il lato della sostenibilità, del design e dell'ergonomia dell'imbarcazione.

Per quanto riguarda la sostenibilità, lo sviluppo di motori ibridi a basso consumo (che rientra nella categoria design rivolto al risparmio energetico), assume un ruolo centrale.

Questo anche perché - come emerge dalle interviste effettuate alle associazioni di categoria locali ${ }^{9}$ - "i nuovi motori, oltre ad incidere dal lato dell'inquinamento in mare, consentono di perseguire un risparmio economico attraverso l'abbattimento dei costi di navigazione".

Particolare attenzione viene rivolta al design dell'imbarcazione, con l'istituzione di centri stile specializzati che garantiscono la massima personalizzazione anche attraverso la consulenza creativa di artisti.

Anche per quanto riguarda l'ergonomia dell'imbarcazione, si sottolinea la ricerca di nuove forme progettuali che consentano da un lato di ottimizzare gli spazi interni e dall'altro di inserire sistemi di stabilizzazione che rendano la navigazione meno turbolenta e più piacevole.

Nel complesso si tratta di processi incrementali d'innovazione che mirano per lo più a politiche di redesign del prodotto (Wever et al., 2008) con l'obiettivo di introdurre graduali miglioramenti per ridurre l'impatto ambientale dei prodotti esistenti.

Tra le imprese terminali, quelle che dimostrano un minor attivismo nei processi di eco-design, risultano essere i produttori di superyacht e megayacht (navi da diporto). Questo dato - come risulta anche dalle interviste ${ }^{10}$ - dipende dal fatto che "il cliente dell'extra lusso non ha bisogno di risparmiare, il core business è l'estrema personalizzazione, quindi il green non è un valore riconosciuto".

In definitiva, per quanto concerne la dimensione comportamentale, la differenza maggiore riguarda le imprese di fase e le imprese terminali.

Le imprese terminali presentano un significativo grado di sustainability orientation - mettendo in campo le innovazioni più significative rivolte alla sostenibilità - mentre le imprese di fase non possono qualificarsi come imprese

9 Intervista a Francesco Buscaglia e Valeria Bertani (Confindustria Pesaro e Urbino) del gennaio 2013

10 Intervista a Moreno Bordoni (CNA Pesaro e Urbino) del 10 gennaio 2013. 
orientate alla sostenibilità. Tale dato è confermato anche dalle interviste effettuate alle associazioni di categoria locali ${ }^{11}$ dei piccoli produttori nautici, "le microimprese di fase sono obbligate dalla crisi a concentrarsi totalmente nella gestione dell'ordinaria attività per cercare di sopravvivere e non hanno le risorse necessarie per effettuare investimenti in ricerca e sviluppo".

Viceversa, le imprese terminali presentano un significativo dinamismo sulle certificazioni ambientali, ma soprattutto sulle politiche orientate allo sviluppo di prodotti di eco-design (3 imprese su 5 hanno già attivato questi processi).

\subsubsection{L'impatto sulla gestione strategica e sulla competitività delle politiche sostenibili}

Dopo aver valutato la dimensione culturale e comportamentale delle imprese, si è cercato di comprendere quali effetti l'orientamento alla sostenibilità possa determinare sulla competitività delle imprese. Agli intervistati è stato chiesto di attribuire alle variabili in figura 6 un punteggio, con scala di valori da 1 a 5 .

L'analisi è stata effettuata su due differenti livelli: il primo attiene alla media generale, il secondo prende in considerazione le sole risposte delle imprese terminali, che sono la tipologia d' impresa che manifesta un livello di orientamento alla sostenibilità più marcato.

Come si può notare, i valori medi risultano essere tendenzialmente bassi.

Fig. 6: Domanda del questionario: qual è l'impatto delle politiche sostenibili sulla competitività d'impresa?

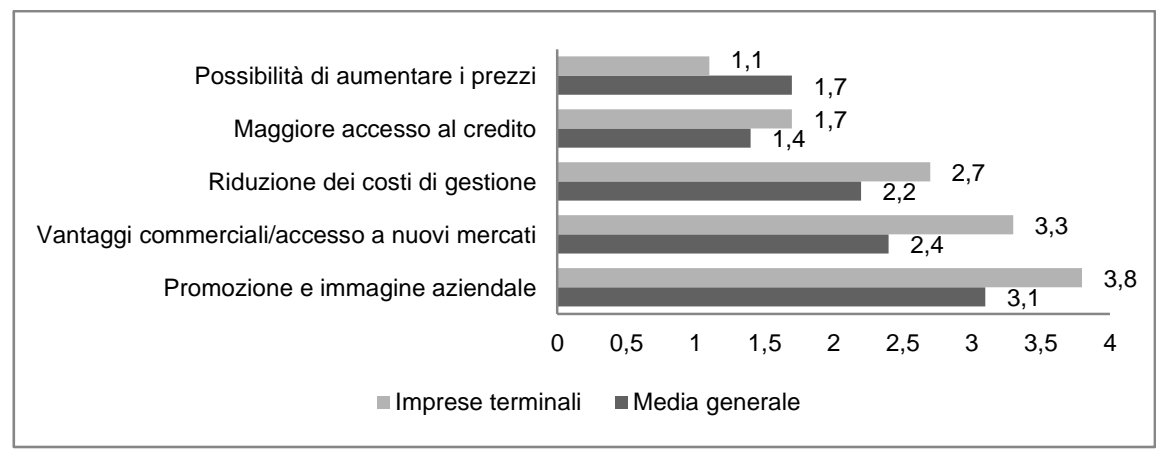

Fonte: nostra elaborazione

Gli indicatori che ottengono i punteggi maggiori sono la promozione dell'immagine aziendale $(3,1)$, il conseguimento di vantaggi commerciali e l'accesso

11 Intervista a Silvana Della Fornace (Confartigianato Pesaro e Urbino) del 14 gennaio 2013. 
a nuovi mercati $(2,4)$. I tre indicatori che ottengono il punteggio minore sono: maggiore accesso al credito $(1,4)$, possibilità di aumentare i prezzi $(1,7)$.

Se confrontiamo i punteggi medi conseguiti dal campione con i punteggi medi delle imprese terminali risulta che le seconde dichiarano di ottenere maggiori benefici rispetto alla media del campione in relazione ai processi commerciali $(3,8$, contro una media di 3,1$)$, alle attività di comunicazione $(3,3$ contro una media di 2,4$)$ ed al contenimento dei costi di gestione (2,7 contro una media di 2,2$)$.

Quanto emerso riflette a nostro avviso l'idea che al momento le politiche di sostenibilità siano concepite dalle imprese terminali del settore soprattutto e comunque in chiave di comunicazione di mercato, con considerazione sostanzialmente marginale delle altri fonti di vantaggio competitivo.

\section{Conclusioni}

Il lavoro parte da una breve ma indicativa osservazione del comparto della nautica da diporto in Italia, dalla quale emerge con chiarezza la perdita di competitività di un sistema che ha subito gli effetti più devastanti della crisi economica globale. Il recupero della competitività delle imprese passa anche attraverso nuovi orientamenti produttivi, maggiormente in linea non solo con la domanda ma anche con le politiche normative dettate dalla necessità di salvaguardare equilibri sociali ed ambientali a livello locale come a livello europeo. In tale prospettiva si inserisce la ricerca empirica avente come ambito geografico la regione Marche, tra le più colpite dalla crisi e dall'andamento negativo del comparto. Lo studio pone l'attenzione sul grado di orientamento alla sostenibilità delle imprese, anzitutto attraverso l'applicazione di un modello di analisi teso a valutarne l'intensità come sintesi tra adesione culturale ai principi della sostenibilità ed effettiva attuazione di scelte aziendali ad essi ispirate.

L'analisi empirica mostra una situazione assai variegata e ricca di evidenze contrastanti. Riguardo la dimensione culturale dell'orientamento alla sostenibilità emerge un buon grado di consapevolezza da parte delle imprese intervistate, siano esse terminali che di fase. I risultati più netti si riscontrano nell'aspetto relativo alla consapevolezza dichiarata e quindi alla disponibilità/propensione ad investire in politiche per la sostenibilità. Tuttavia tale consapevolezza si fonda maggiormente sull'idea che investire in politiche sostenibili "produce benefici per il futuro" e non tanto perché "è un valore riconosciuto dal mercato".

Per quanto riguarda la dimensione comportamentale, ovvero l'effettiva pratica di politiche orientate alla sostenibilità, si è riscontrata una disomogeneità tra le differenti tipologie d'imprese intervistate. Le imprese terminali dedicano adeguata attenzione alle attività di certificazione ambientale e alla progettazione di ecoprodotti, mentre le imprese di fase non adottano comportamenti particolarmente orientati alla sostenibilità, dimostrando come la dimensione culturale non rappresenti un prerequisito sufficiente ad un effettivo impegno in politiche sostenibili. Tale tendenza pare motivata da diversi fattori, tra i quali assume un ruolo centrale la piccola dimensione, spesso accompagnata da scarsità di risorse 
finanziarie e manageriali, oltre che dall'assenza di un contatto diretto con il mercato. Ciò rende difficoltoso intravedere le opportunità legate a condotte orientate alla sostenibilità.

Il punto cruciale è che queste piccole imprese - essendo spesso prive delle risorse necessarie sia in termini di capacità finanziarie che di competenze - al fine di attuare una strategia di questo tipo dovrebbero percorrere la strada della collaborazione (Pencarelli, 1995; Penco, 2010). È infatti solo attraverso l'integrazione e la collaborazione che il sistema di piccole imprese potrà acquisire le risorse e le capacità necessarie per conquistare e controllare i fattori critici di successo del settore, come la sostenibilità (Bortoluzzi e Tracogna, 2011). A questo riguardo si sono messi recentemente in campo alcuni tentativi di realizzare contratti di rete nel comparto, per ora con risultati incerti e non quantificabili.

Per quanto concerne l'impatto delle politiche sostenibili sulla competitività delle imprese, emerge che per gli intervistati queste incidono significativamente solo sulle variabili che riguardano le politiche di comunicazione volte a rafforzare l'immagine e la reputazione aziendale e, in misura inferiore, l'accesso a nuovi segmenti di domanda, mentre non sembrano essere considerate requisiti cruciali per il successo a livello di gestione dei costi e dei prezzi. Come era facile prevedere, risulta inoltre che il peso maggiore alla variabile reputazionale e all'accesso a nuovi mercati è attribuito dalle aziende terminali, che hanno assegnato un punteggio di 3,8 all'immagine - contro una media di 3,1 - ed un punteggio di 3,3 (appena sopra lo score medio della scala di Likert) alla variabile commerciale - contro una media di appena il 2,4.

In questo modo le imprese del settore nautico marchigiano sembrano trascurare un tema di crescente importanza quale potrà essere a nostro avviso quello della possibile riduzione dei costi di produzione e di utilizzo delle imbarcazioni, nel quadro dell'emergente esigenza di maggiore accettabilità della nautica nell'ambito delle città portuali (Pencarelli e Forlani, 2007; Cherubini e Nastasi, 2006; Quagli, 2009), ove le comunità s'interrogano sugli effetti inquinanti del settore, premendo per una maggiore attenzione agli equilibri eco-sistemici. Inoltre non si tiene sufficientemente conto della presenza nel mercato mondiale di un numero crescente di utilizzatori sensibili ai temi della sostenibilità e dell'eco-design, specie laddove riferiti alle tematiche dell'ergonomia e della facilità di fruizione per le persone portatrici di disabilità legate allo stato di salute, all'età ed ad altre forme di possibile handicap. Vanno inoltre colti i segnali provenienti dai regolatori mondiali ed europei, che probabilmente metteranno in campo norme sempre più vincolanti per $\mathrm{i}$ produttori, orientandoli verso processi guidati dall'eco-design.

Per questo le future scelte strategiche delle imprese della nautica da diporto non potranno prescindere dall'attivare processi d'innovazione tecnologica guidati dal tema della sostenibilità, inteso sia nella dimensione ecologica ed ambientale, che in quello sociale ed economico. La sostenibilità dei prodotti e dei processi produttivi, dunque, come nuovo ed importante fattore critico di successo del settore, da perseguire anche come veicolo di differenziazione in grado di determinare vantaggi competitivi da prima mossa, aggiungendosi ai tradizionali fattori di competitività 
del settore, come la tecnologia, l'estetica, la funzionalità e la performance e la dimensione del lusso delle imbarcazioni.

Per questo si rende necessario - in un ottica di lungo periodo - che le imprese terminali coinvolgano tutta la tradizionale catena estesa del valore (sistema del valore), trasformandola in una "Green supply chain", imponendo l'applicazione di standard produttivi di filiera sostenibili (Figura 7).

Fig. 7: Le politiche di eco-design come output di processi innovativi delle imprese della nautica da diporto sustainability oriented
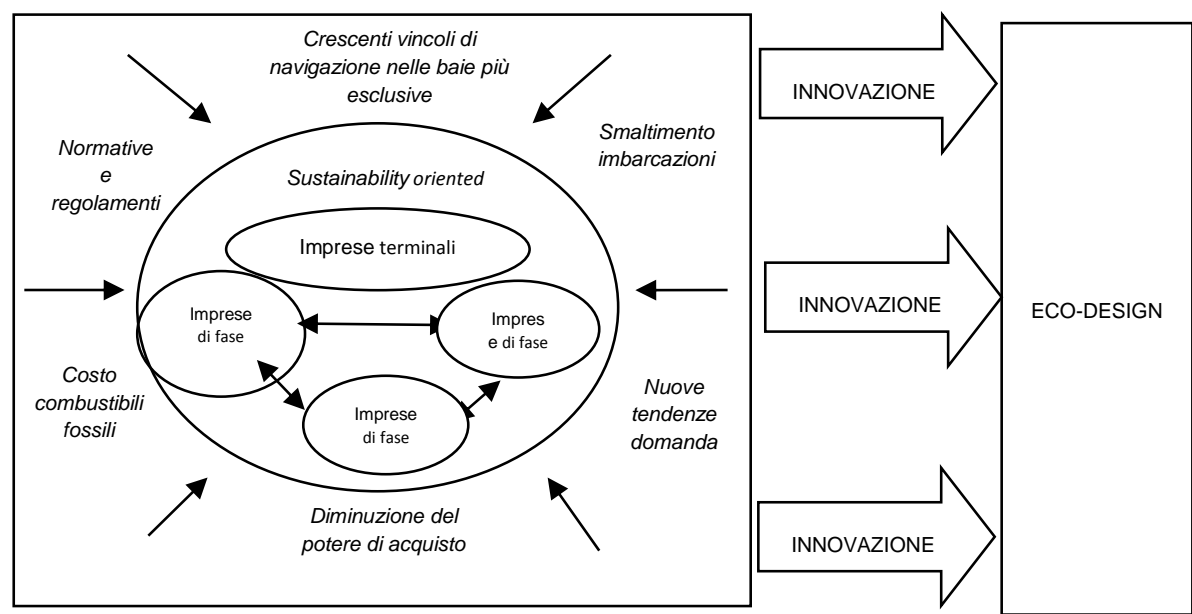

Fonte: nostra elaborazione

Tali standard e politiche operative, stimolate - se non obbligate - da fattori esterni alle imprese, esercitano una influenza diretta sul comportamento strategico. Esempi, in tal senso, possono essere le normative e i regolamenti sulla produzione, l'aumento del costo dei combustibili fossili, il problema dello smaltimento delle imbarcazioni alla fine del ciclo di vita, ecc. Tali input esterni, congiuntamente a fattori interni all'impresa e relativi alla componente culturale, stimolano innovazioni nei processi produttivi, classificabili all'interno della filosofia dell'eco-design.

La sfida riguarda dunque non solo le aziende terminali, ma anche quelle di fase, sebbene non possa sfuggire che non tutte le piccole imprese terziste coinvolte nel sistema saranno in grado di inserirsi in circuiti relazionali di questo tipo; questo dipenderà soprattutto dalla qualità delle risorse e delle competenze interne, nonché dall'abilità di padroneggiare i linguaggio delle reti (Pencarelli, 1995).

Su questi temi la ricerca è chiamata a ulteriori sentieri di approfondimento, a partire dalla comprensione delle effettive dinamiche della domanda e delle regolazioni di mercato, fino ad approdare alle possibili interrelazioni e sinergie competitive che il sistema nautico da diporto regionale saprà intrattenere con altri 
sistemi e comparti in un ottica di nuovo "sistema del valore allargato" (Ferrero e Fortezza, 2005), orientato alla sostenibilità.

\section{Bibliografia}

ANTONELLI G., BISCHI G.I., VIGANO’ E. (2005), La sostenibilità nel settore della pesca. Modelli, politiche, esperienze in un'area del litorale romagnolo-marchigiano, FrancoAngeli, Milano.

BENEVOLO C. (2010), "Turismo nautico - Una sfida per il destination management", Rivista di scienza per il turismo, n. 3, pp. 105-129.

BENEVOLO C. (2011), "Problematiche di sostenibilità nell'ambito del turismo nautico in Italia", Electronic journal of management, n. 2, pp. 1-17.

BORTOLUZZI G., TRACOGNA A. (2011), "Processi di innovazione e fattori di competitività nei cluster internazionali della nautica", Sinergie, n. 84, pp. 89-118.

BRUNI M., CARCANO L. (2009), La nautica italiana - Modelli di business e fattori di competitività, Egea, Milano.

CHERUBINI S., NASTASI T. (2006), Il marketing della nautica da diporto: dal prodotto al sistema nautico integrato, Nautica Editrice, Roma.

CORBETTA P. (1999), Metodologia e tecniche della ricerca sociale, Il Mulino, Bologna.

DI MARIA E., BETTIOL M. (2009), "Management dell'innovazione", in Di Bernardo B., Gandolfi V., Tunisini A. (a cura di), Economia e management delle imprese, Hoepli, Milano.

FERRANDO M., MORRA M.C., MAZZUCCHELLI A. (2014), "Il progetto Innautic", in Di Gregorio A. (a cura di), L'esercizio di foresight. Una risorsa di conoscenza per la competitività dei territori, Franco Angeli, Milano.

FERRERO G., FORTEZZA F. (2005), "Processi di creazione del valore e sistemi locali di imprese nel settore della nautica da diporto", Small Business, n. 3, pp. 73-107.

FONDAZIONE SYMBOLA \& UNIOCAMERE (2010), "Innovazione ed efficienza nella competitività della nautica", Rapporto Green Italy, Roma.

FONDAZIONE SYMBOLA \& UNIOCAMERE (2011), "L'economia verde sfida la crisi", Rapporto Green Italy, Roma.

FONDAZIONE SYMBOLA \& UNIOCAMERE (2012), "La nautica italiana. Reti territori e sostenibilità, Rapporto Green Italy, Roma.

FORTEZZA F. (2008), Processi strategici e di marketing nel settore della nautica da diporto, Franco Angeli, Milano.

GARDNER H. (2007), "Una voce fuori dal coro", Harvard Business Review Italia, n. 4, pp. $1-8$.

GRANDE S. (2009), "Smaltimento e dismissione di imbarcazioni a fine vita commerciale utile", in Bruni M., Carcano L. (a cura di), La nautica italiana - Modelli di business e fattori di competitività, Egea, Milano.

KOENIG G.K. (1983), “Design: rivoluzione, evoluzione o involuzione?”, Ottagono, n. 68, pp. 24.

LAZZARETTI L., CAPONE F. (2009), "Industrial district effects and innovation in the Tuscan shipbuilding industry", IERMB Working Paper in Economics, n. 09.03.

MARIANI A., VIGANO' E. (2002), Il sistema agro-alimentare dell'Unione Europea. Economia e politiche, Carocci Editore, Roma.

MOSCHINI R. (2009), Aree protette e nautica sostenibile, ETS, Pisa. 
NOBILI E. (2012), “I sistemi nautici tra crisi e opportunità. L'esperienze della provincia di Pesaro e Urbino", in Quattrociocchi B., (a cura di), Economia del mare e processi d'internazionalizzazione. Verso una rete transazionale per il Mediterraneo, Franco Angeli, Milano.

PENCARELLI T. (1995), Piccola impresa, alleanze strategiche ed integrazione europea, Aspi/Ins-Edit, Genova.

PENCARELLI T., FORLANI F. (2007), Turismo nautico e porti turistici. Quali prospettive nelle Marche?, Quattroventi, Urbino.

PENCARELLI T., SPLENDIANI S. (2012), "Imprese turistiche e sostenibilità: evidenze empiriche nella provincia di Pesaro e Urbino”, XXXIII Annual Conference of Regional Science (A.I.S.Re.), Roma, Settembre 13-15.

PENCO L. (2010), "Dai sistemi locali ai network de-territorializzati: verso i meta-distretti e le reti tra distretti", Sinergie, n. 83, pp. 3-29

PROGETTO ENA (2012), "Report 03_ricerca fabbisogni conoscitivi_analisi del questionario_imprese", www.progettoena.it.

QUAGLI A. (2009) (a cura di), Analisi gestionale dei porti turistici nella nautica da diporto. Il caso di Imperia, Franco Angeli, Milano.

QUILDON L.F., PUN K.F. (2011), "Development of a Value Creation Programme in the YachtingServices Cluster: A Value Management Approach”, The West Indian Journal of Engineering, n. 33, pp. 71-77.

RAPPORTO GREENITALY (2010), Innovazione ed efficienza nella competitività della nautica, Fondazione Symbola \& Unioncamere, Viareggio.

RAPPORTO GREENITALY (2011), L'economia verde sfida la crisi, Fondazione Symbola \& Unioncamere, Gaeta.

RAPPORTO GREENITALY (2012), La nautica italiana. Reti territori e sostenibilità, Fondazione Symbola \& Unioncamere, Roma.

TAMBORRINI P. (2009), design sostenibile. oggetti, sistemi e comportamenti, Electa, Milano.

THACKARA J. (2008), In the bubble: design per un futuro sostenibile, Allemandi, Torino.

TOLA A. (2013) (a cura di), Il settore della nautica nel Nord Sardegna. Innovazione tecnologica, sviluppo competitivo e dinamica di crescita delle imprese, Franco Angeli, Milano.

TRACOGNA A. (2007), I cluster del mare: nautica da diporto e cantieristica navale in Friuli Venezia Giulia, Franco Angeli, Milano.

TRACOGNA A. (2010), I cluster del mare: analisi comparativa, forme di governance $e$ politiche di sviluppo, Franco Angeli, Milano.

UCINA (2008, 2009, 2010, 2011, 2012), La nautica in cifre, Genova

VEZZOLI C., MANZINI E. (2007), Design per la sostenibilità ambientale, Zanichelli Editore, Bologna.

WEVER R., BOKS C., BAKKER C.A. (2008), "Sustainability within Product Portfolio Management", Sustainable Innovation Conference Malmö, Ottobre 27-28, pp. 219 227.

YIN R.K. (2009), Case study research: design and methods, Sage publications, Beverly Hills.

ZUCCON G. (2009), "Il design per la crisi", in Bruni M., Carcano L. (a cura di.), La nautica italiana. Modelli di business e fattori di competitività, Egea, Milano. 